\title{
Uso de guia tridimensional personalizado no preparo do orifício do pedículo piloto em deformidades da coluna vertebral
}

\section{Use of a Customized Three-dimensional Guide in Preparing the Pilot Pedicle Hole in Spinal Deformities}

\author{
Kelsen de Oliveira Teixeira ${ }^{10}$ Thiago Dantas Matos ${ }^{10}$ Rodrigo Barra Caiado Fleury ${ }^{10}$ \\ Herton Rodrigo Tavares Costa ${ }^{20}$ Helton Luiz Aparecido Defino ${ }^{30}$ \\ 1 Programa de Pós-Graduação, Faculdade de Medicina de Ribeirão \\ Endereço para correspondência Kelsen de Oliveira Teixeira, Master, \\ Preto, Universidade de São Paulo, Ribeirão Preto, SP, Brasil \\ Rua Dr. Amâncio de Carvalho, 86. Ed. Tita Salzano, Ap. 406, P.O, São \\ ${ }^{2}$ Hospital das Clínicas, Faculdade de Medicina de Ribeirão Preto, \\ Paulo, SP, 04012-080, Brasil (e-mail: kelsenteixeira@gmail.com).
}

Universidade de São Paulo, Ribeirão Preto, SP, Brasil

${ }^{3}$ Departamento de Ortopedia e Traumatologia, Faculdade de Medicina de

Ribeirão Preto, Universidade de São Paulo, Ribeirão Preto, SP, Brasil

Rev Bras Ortop 2022;57(3):375-383.

\section{Resumo}

\section{Palavras-chave}

- fraturas ósseas

- anormalidades congênitas

- coluna vertebral

- vértebras torácicas
Objetivo O presente estudo teve como objetivo desenvolver e avaliar a utilização de guias personalizadas em pacientes submetidos a cirurgia para correção de deformidades vertebrais com sistema de fixação pedicular.

Métodos Quatro pacientes com deformidade espinhal (três casos de escoliose idiopática e um caso de cifoescoliose congênita) foram submetidos a tratamento cirúrgico corretivo com sistema de fixação pedicular. Protótipos de guias tridimensionais foram desenvolvidos e avaliados quanto à viabilidade técnica, precisão e exposição à radiação.

Resultados $\mathrm{O}$ presente estudo incluiu 85 pedículos vertebrais submetidos à inserção de parafusos pediculares na coluna torácica $(65,8 \%)$ e na coluna lombar $(34,2 \%)$. A viabilidade técnica foi positiva em 46 pedículos vertebrais (54,1\%), sendo 25 torácicos (54\%) e 21 lombares (46\%). A viabilidade técnica foi negativa em 39 pedículos (45,9\%), sendo 31 torácicos (79,5\%) e 8 lombares (20,5\%). Quanto à precisão, 36 parafusos foram centralizados (78,2\%), sendo 17 na coluna torácica $(36,9 \%)$ e 19 na coluna lombar (41,3\%). O mau posicionamento foi observado em 10 parafusos $(21,7 \%)$, sendo 8 na coluna torácica $(17,4 \%)$ e 2 na coluna lombar (4,3\%). A radiação média registrada nos procedimentos cirúrgicos foi de $5,17 \pm 0,72 \mathrm{mSv}$, e o tempo total de uso da fluoroscopia em cada cirurgia variou de 180,3 a 207,2 segundos.
Trabalho desenvolvido no Departamento de Ortopedia e Traumatologia, Hospital das Clínicas, Faculdade de Medicina de Ribeirão Preto, Universidade de São Paulo, Ribeirão Preto, SP, Brasil. recebido

31 de Julho de 2020

aceito

02 de Outubro de 2020

Publicado on-line

Agosto 13, 2021
DOI https://doi.org/

$10.1055 / \mathrm{s}-0041-1724074$ ISSN $0102-3616$. (c) 2021. Sociedade Brasileira de Ortopedia e Traumatologia. All rights reserved.

This is an open access article published by Thieme under the terms of the Creative Commons Attribution-NonDerivative-NonCommercial-License, permitting copying and reproduction so long as the original work is given appropriate credit. Contents may not be used for commercial purposes, or adapted, remixed, transformed or built upon. (https://creativecommons.org/ licenses/by-nc-nd/4.0/)

Thieme Revinter Publicações Ltda., Rua do Matoso 170, Rio de Janeiro, RJ, CEP 20270-135, Brazil 


Abstract


Keywords
- fractures, bone
- congenital
abnormalities
- spine
- thoracic vertebrae

Conclusão Os protótipos de guias personalizadas permitiram o preparo seguro do orifício piloto nos pedículos vertebrais em casos de deformidade, com maior precisão e menor exposição intraoperatória à radiação.

Objective The present study aimed to develop and evaluate the use of customized guides in patients undergoing surgery to correct vertebral deformity with a pedicular fixation system.

Methods Four patients with spinal deformity (three with idiopathic scoliosis and one with congenital kyphoscoliosis) underwent surgical treatment to correct the deformity with a pedicular fixation system. Prototypes of 3D cost guides were developed and evaluated using technical feasibility, accuracy, and radiation exposure.

Results The present study included 85 vertebral pedicles in which pedicle screws were inserted into the thoracic spine (65.8\%) and into the lumbar spine (34.2\%). Technical viability was positive in 46 vertebral pedicles (54.1\%), with 25 thoracic (54\%) and 21 lumbar (46\%). Technical viability was negative in 39 pedicles (45.9\%), 31 of which were thoracic (79.5\%), and 8 were lumbar (20.5\%). In assessing accuracy, 36 screws were centralized (78.2\%), of which 17 were in the thoracic $(36.9 \%)$ and 19 in the lumbar spine (41.3\%). Malposition was observed in 10 screws (21.7\%), of which 8 were in the thoracic (17.4\%) and 2 in the lumbar spine (4.3\%). The average radiation record used in the surgical procedures was of $5.17 \pm 0.72 \mathrm{mSv}$, and the total time of use of fluoroscopy in each surgery ranged from 180.3 to 207.2 seconds.

Conclusion The customized guide prototypes allowed the safe preparation of the pilot orifice of the vertebral pedicles in patients with deformities with improved accuracy and reduced intraoperative radiation.

\section{Introdução}

Os sistemas para correção de deformidades da coluna vertebral usam principalmente parafusos pediculares para ancoragem posterior. ${ }^{1}$ Esses sistemas permitem a correção tridimensional (3D) das deformidades, dando estabilidade suficiente para evitar a imobilização pós-operatória., 2,3 Além disso, este sistema permite a correção ainda mais significativa de deformidades, principalmente em comparação com sistemas híbridos ou com ganchos. ${ }^{4-7}$ No entanto, os sistemas de fixação pedicular têm algumas desvantagens, em especial as complicações decorrentes do posicionamento incorreto do parafuso dentro do pedículo e exposição do cirurgião a radiação. ${ }^{8} \mathrm{O}$ posicionamento incorreto dos parafusos pediculares é mais comum em pacientes com deformidades devidas a alterações anatômicas das vértebras e sua posição 3D. 9,10 $^{9,10}$

A precisão média de colocação dos parafusos pediculares à mão livre ou sob fluoroscopia e com auxílio da navegação é de $85,1 \%$ e $95,5 \%$, respectivamente. ${ }^{11-14}$ A fluoroscopia é bastante usada para auxiliar a inserção de parafusos pediculares. ${ }^{14}$ Durante a fluoroscopia, porém, a exposição do cirurgião a radiação é 10 a 12 vezes maior do que em outros procedimentos fluoroscópicos em segmentos fora da coluna vertebral. ${ }^{15,16}$

Novas alternativas foram desenvolvidas para melhorar a precisão e reduzir a exposição a radiação, com ênfase em guias personalizadas. ${ }^{17-19}$ As vantagens da utilização de uma guia personalizada (em especial seu baixo custo) nos motivaram a realizar um projeto de desenvolvimento de um protótipo.

Dessa forma, o presente estudo teve como objetivo desenvolver e avaliar a utilização de guias personalizadas em pacientes submetidos à cirurgia para correção de deformidade vertebral com sistema de fixação pedicular. Essas guias são confeccionadas com impressão 3D a partir de modelos vertebrais e desenvolvidas para auxiliar o preparo do orifício piloto no pedículo vertebral.

\section{Métodos}

O Comitê de Ética em Pesquisa aprovou o presente estudo (número do protocolo: 3.365.105).

0 presente estudo foi realizado em quatro pacientes com deformidades da coluna vertebral submetidos a tratamento cirúrgico com sistema de fixação pedicular.

Os dados demográficos dos pacientes são mostrados na - Tabela 1. Três pacientes tinham escoliose idiopática e uma paciente apresentava cifoescoliose. Todas as pacientes eram do sexo feminino, com idade entre 11 e 17 anos (média $=15$ anos).

Um conjunto de guias 3D foi feito para cada paciente. Uma guia foi criada para cada vértebra que seria submetida à fixação pedicular. Além das guias, um modelo da coluna vertebral também foi confeccionado para auxiliar a orientação 3D das estruturas vertebrais (-Fig. 1). 
Tabela 1 Dados demográficos dos pacientes avaliados no estudo.

\begin{tabular}{|l|l|l|l|l|l|}
\hline & Idade (anos) & Gênero & Deformidade & Níveis $^{*}$ & Ângulo de Cobb \\
\hline Paciente $\mathbf{1}$ & 16 & Feminino & AIS & T10-L4 & $65,8^{\circ}$ \\
\hline Paciente $\mathbf{2}$ & 17 & Feminino & JIS & T3-L3 & $68,1^{\circ}$ \\
\hline Paciente 3 & 11 & Feminino & Cifoescoliose congênita & T8-L2 & $57,5^{\circ}$ (escoliose) $/ 87,3^{\circ}($ cifose) \\
\hline Paciente 4 & 15 & Feminino & JIS & T6-L2 & $64,1^{\circ}$ \\
\hline
\end{tabular}

Abreviações: AIS, escoliose idiopática adolescente; JIS, escoliose idiopática juvenil. *níveis de extensão da curva escoliótica.

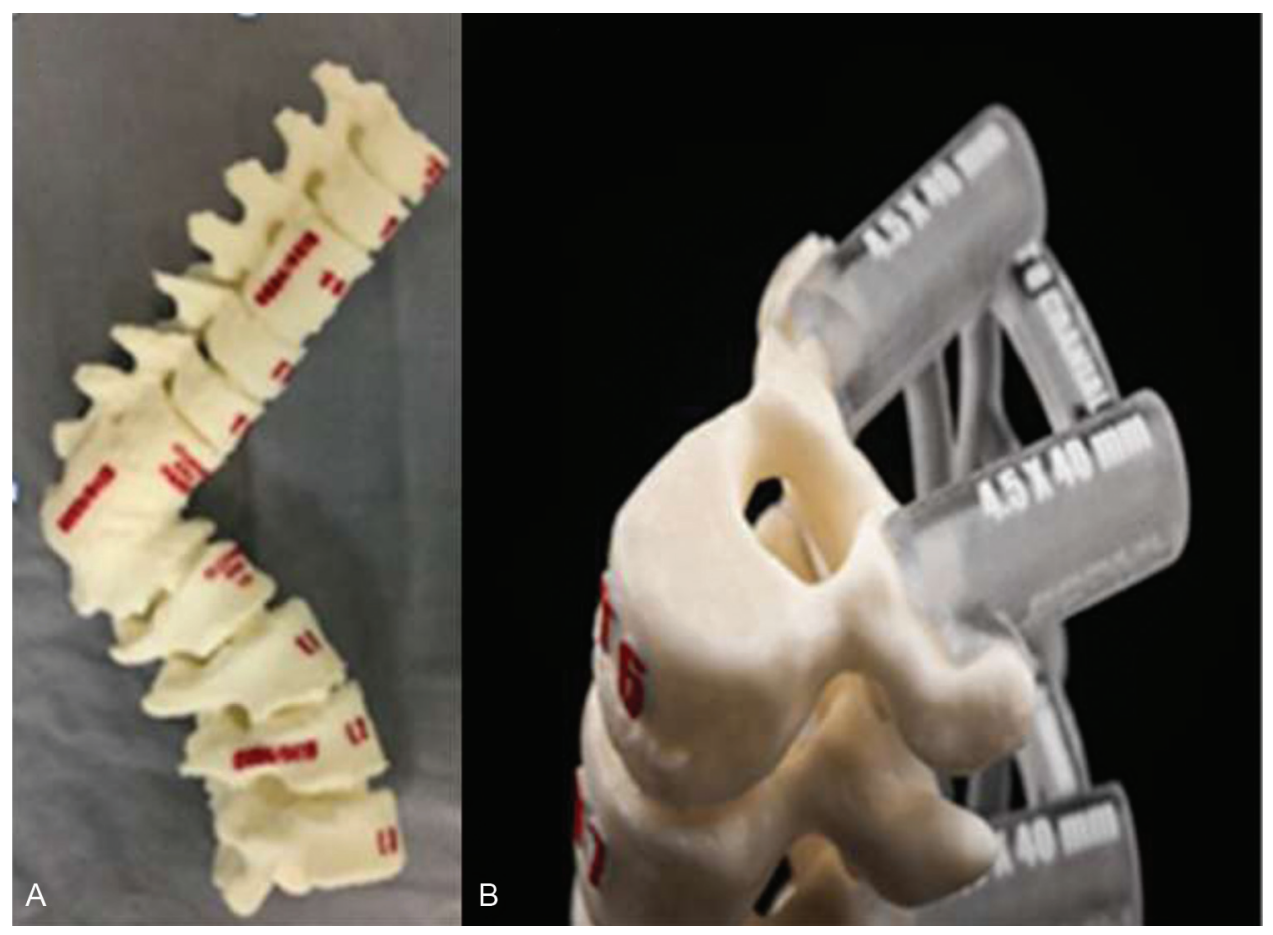

Fig. 1 Imagens ilustrativas. (A) Fotografia do modelo da coluna de um paciente com deformidade congênita; (B) Fotografia da guia cirúrgica fixada ao modelo na posição de preparo do orifício piloto.

As guias 3D foram construídas a partir dos achados da tomografia computadorizada (TC) pré-operatória cobrindo a extensão do segmento vertebral que receberia os parafusos pediculares. A TC foi padronizada em cortes $\leq 1$ milímetro para aumento da precisão da reconstrução anatômica da superfície óssea.

O planejamento pré-operatório para determinação bilateral, em cada vértebra, do posicionamento do parafuso no interior do pedículo vertebral, sua angulação e comprimento usou a análise anatômica 3D (ATA) do software (Materialise Brasil. São Paulo, SP, Brasil). O cirurgião orientou a posição, a angulação e o comprimento do parafuso pedicular a ser usado (-Fig. 2).

As guias foram confeccionadas com resina sintética biocompatível, não biodegradável, e submetidas à esterilização em temperatura de $50^{\circ} \mathrm{C}$ em aparelho Sterrad (Medsteril, Água Branca, São Paulo, SP, Brasil). Uma guia específica foi criada para cada vértebra usando uma impressora 3D. Cada guia, feita para uma determinada vértebra, consistia em duas partes cilíndricas que orientavam o ponto de entrada e preparo do orifício piloto do pedículo vertebral por meio da colocação de instrumentos em seu interior (-Fig. 3).
Durante o procedimento cirúrgico, as guias foram acopladas a cada vértebra por meio de seu encaixe no processo espinhoso e da oposição da superfície das guias no ponto correspondente à projeção do pedículo vertebral no dorso vertebral (-Fig. 4)

Com a guia posicionada e estabilizada, o ponto de entrada no pedículo vertebral foi determinado pela introdução do instrumento apropriado em seu interior. Em seguida, o orifício piloto foi preparado com as sondas colocadas em seu interior, seguido de punção e verificação das paredes do pedículo vertebral antes da inserção dos parafusos.

Os seguintes parâmetros foram utilizados para avaliação do uso das guias: viabilidade técnica, precisão e exposição à radiação.

O desempenho técnico da guia em sua utilização para o fim pretendido foi analisado e classificado como positivo ou negativo. Portanto, a viabilidade técnica foi considerada positiva quando a guia pode ser utilizada da maneira desejada. Por outro lado, a viabilidade técnica foi considerada negativa quando a guia não pôde ser utilizada ou não atingiu os objetivos desejados (ajuste inadequado da guia nos elementos vertebrais posteriores, ponto de perfuração sem correlação às referências 

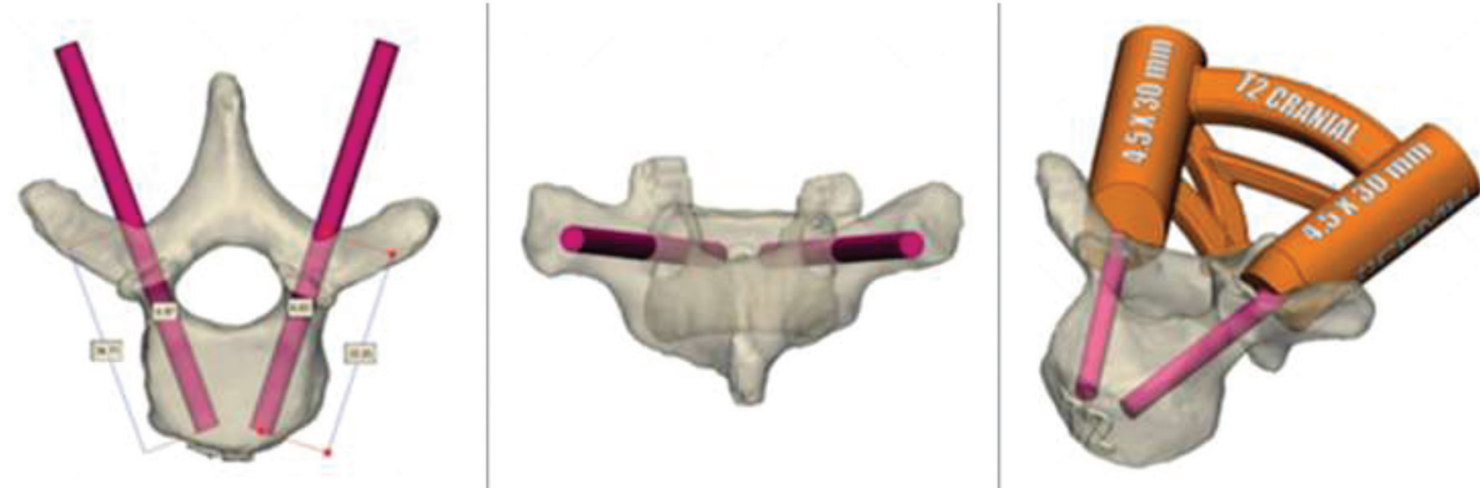

Fig. 2 Fotografia da análise anatômica tridimensional pré-operatória em diferentes ângulos com simulação da posição dos parafusos pediculares e o encaixe da guia cirúrgica na região posterior da vértebra correspondente.

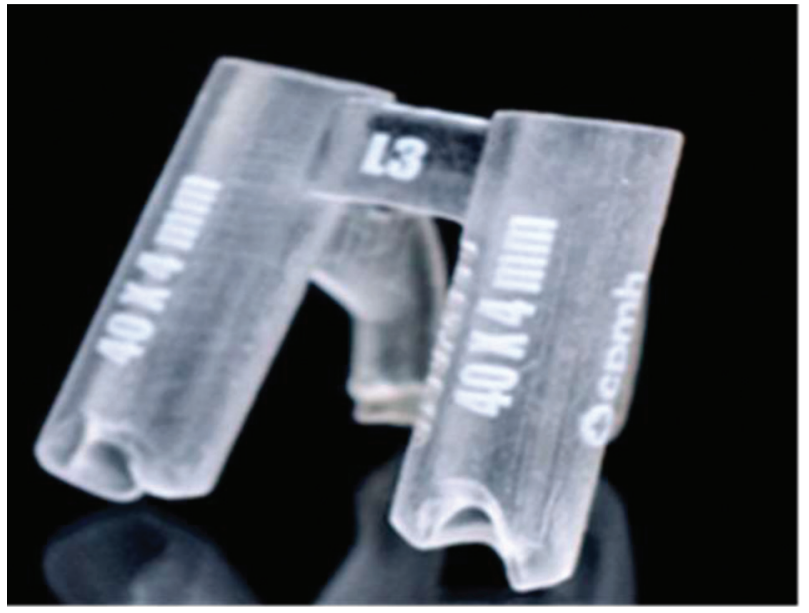

Fig. 3 Fotografia da guia cirúrgica para uma vértebra lombar.

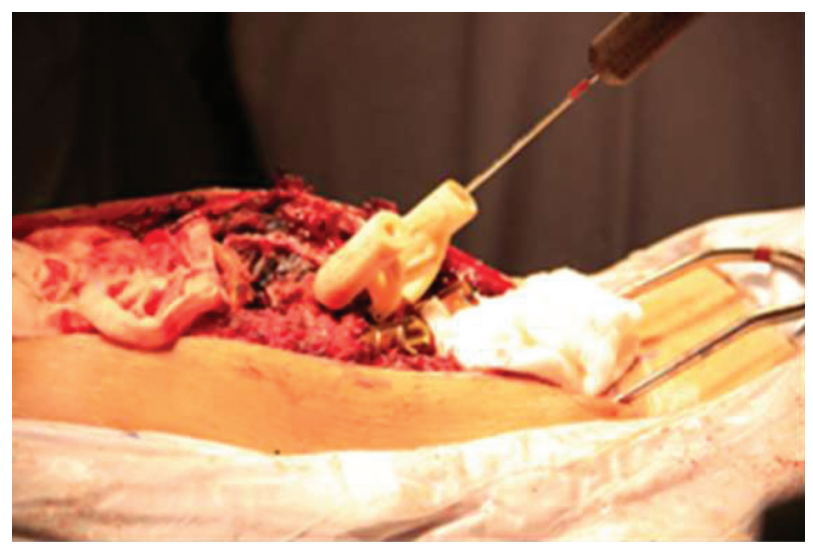

Fig. 4 Imagem intraoperatória de uma guia cirúrgica posicionada na região vertebral posterior, com instrumento acoplado para preparo do orifício piloto.

anatômicas, quebra da guia durante o uso, problemas no acoplamento do instrumental cirúrgico à guia, inadequação do orifício piloto à verificação das paredes do pedículo ou à fluoroscopia).

A precisão foi avaliada no período pós-operatório por meio de TC. O parafuso pedicular foi considerado bem posicionado quando centralizado no pedículo vertebral, mantendo a integridade da parede lateral e da parede medial do pedículo vertebral. A violação da parede lateral ou medial do pedículo vertebral fez com que o parafuso fosse considerado mal posicionado.

A exposição à radiação intraoperatória foi determinada pelo tempo total de uso da fluoroscopia e sua dose.

O teste não paramétrico de Mann-Whitney foi utilizado para análise dos resultados, e o nível de significância foi estabelecido em $p \leq 0,05$.

\section{Resultados}

Avaliamos o conjunto total de 85 pedículos vertebrais (56 torácicos e 29 lombares) submetidos a colocação de parafusos pediculares.

A viabilidade técnica foi positiva em 46 pedículos vertebrais (54,1\%), dos quais 25 eram torácicos (54\%) e 21 lombares (46\%). A viabilidade 8 foi negativa em 39 pedículos (45,9\%), dos quais 31 eram torácicos (79,5\%) e oito lombares (20,5\%). A viabilidade técnica negativa foi causada por diversos fatores, como encaixe inadequado da guia nos elementos vertebrais posteriores (10 pedículos $[11,7 \%]$ ), ponto de perfuração sem correlação às referências anatômicas (23 pedículos [27\%]), quebra da guia durante o uso, problemas no acoplamento dos instrumentos cirúrgicos à guia (2 pedículos [2,5\%]) e inadequação do orifício piloto à verificação das paredes do pedículo ou fluoroscopia (4 pedículos $[4,7 \%]$ ). A viabilidade técnica negativa estava diretamente relacionada às etapas de desenvolvimento do protótipo de guia personalizada e foi reduzida pelo aprimoramento do protótipo.

A avaliação da precisão dos parafusos pediculares em que o orifício piloto foi confeccionado com auxílio da guia mostrou que 36 parafusos estavam centralizados (78,2\%), sendo 17 na coluna torácica (36,9\%) e 19 na coluna lombar (41,3\%).

Em 10 pedículos $(21,7 \%)$, os parafusos não estavam centralizados como estabelecido no planejamento pré-operatório; 6 pedículos (13\%) violaram a parede lateral e quatro $(4,3 \%)$, a parede medial. A precisão dos parafusos na coluna torácica e a concavidade foram menores em relação aos outros segmentos vertebrais.

Aqui, o posicionamento dos parafusos foi predominante na coluna torácica e superior ao grupo de pedículos vertebrais nos quais a guia não pode ser usada. 0 preparo do 
Tabela 2 Análise da posição dos parafusos pediculados com ou sem guias

\begin{tabular}{|c|c|c|c|c|c|c|}
\hline & Com guia & & & Sem guia & & \\
\hline & Violação & & Central & Violação & & Central \\
\hline & Cortical medial & Cortical lateral & & Cortical medial & Cortical lateral & \\
\hline $\mathrm{T} 2$ & & & & 1 & 1 & \\
\hline T3 & & & & & & 1 \\
\hline T4 & & 1 & & 2 & 1 & \\
\hline T5 & & & 2 & & 2 & 2 \\
\hline T6 & & & 2 & 2 & 1 & 1 \\
\hline $\mathrm{T} 7$ & & & & 2 & 1 & 1 \\
\hline T8 & 2 & & 2 & 1 & & 1 \\
\hline T9 & 1 & 1 & 2 & 1 & 1 & 2 \\
\hline T10 & 1 & & 1 & & 1 & 3 \\
\hline T11 & & 1 & 3 & 1 & 1 & 1 \\
\hline $\mathrm{T} 12$ & & 1 & 5 & & & \\
\hline
\end{tabular}

orifício piloto com o auxílio do modelo apresentou maior precisão.

$\mathrm{O}$ parafuso não estava centralizado em 10 pedículos $(21,7 \%)$, sendo 8 na coluna torácica $(17,4 \%)$ e 2 na coluna lombar (4,3\%). A ruptura da parede lateral foi observada em 6 pedículos (13\%), 4 torácicos $(8,7 \%)$ e 2 lombares $(4,3 \%)$. A ruptura da parede medial foi observada em 4 pedículos $(8,7 \%)$, todos na coluna torácica.

Nos 39 pedículos cujos orifícios pilotos foram confeccionados sem auxílio da guia, os parafusos estavam centralizados em 19 (48,7\%), 12 na coluna torácica (30,8\%) e 7 na coluna lombar (17,9\%). O mau posicionamento foi observado em 20 parafusos (51,3\%), 18 na coluna torácica (46,2\%) e 2 na coluna lombar (5,1\%). A ruptura da parede lateral foi observada em 9 pedículos (23\%), todos torácicos. A separação da parede medial foi observada em 11 pedículos (28,2\%), sendo 10 torácicos $(25,7 \%)$ e 1 lombar (2,5\%).
A precisão do conjunto de pedículos nos quais o orifício piloto foi preparado com e sem guia é comparada na - Tabela 2 e na -Fig. 5. A maior eficiência foi relacionada à utilização de guias nos pedículos das vértebras lombares $(p<0,05)$. Por outro lado, nos pedículos da coluna torácica e no conjunto formado por todos os pedículos, não houve diferença estatística quanto à precisão (-Fig. 6). Deve-se considerar que a não utilização das guias de perfuração estava relacionada à viabilidade técnica negativa, e que a visualização intraoperatória do modelo auxiliou na confecção do orifício piloto.

A viabilidade técnica geral teve significância estatística $(p=0,0089)$ ( - Fig. 7) e aumentou de forma gradual após os procedimentos cirúrgicos. $\mathrm{O}$ aperfeiçoamento dos protótipos ( - Fig. 8) tem sido de grande ajuda na correção de deformidades complexas graves (-Fig. $\mathbf{9}$ ).

A exposição à radiação intraoperatória variou de 4,35 milisieverts (mSv) a 6,32 mSv (média $=5,17 \pm 0,72$ ) e o

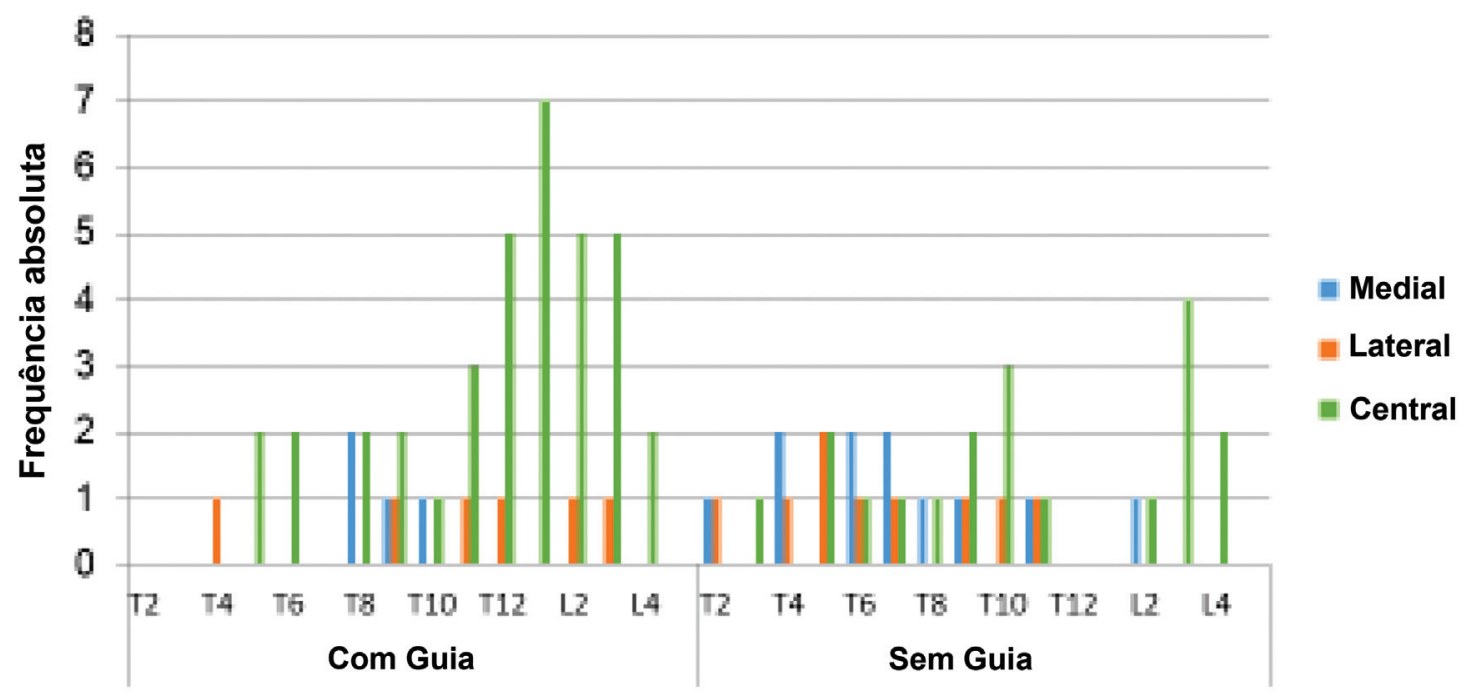

Fig. 5 Comparação da precisão dos parafusos pediculares em nível torácico e lombar em frequência absoluta (número de pedículos) após o posicionamento com e sem auxílio de guias cirúrgicas. 

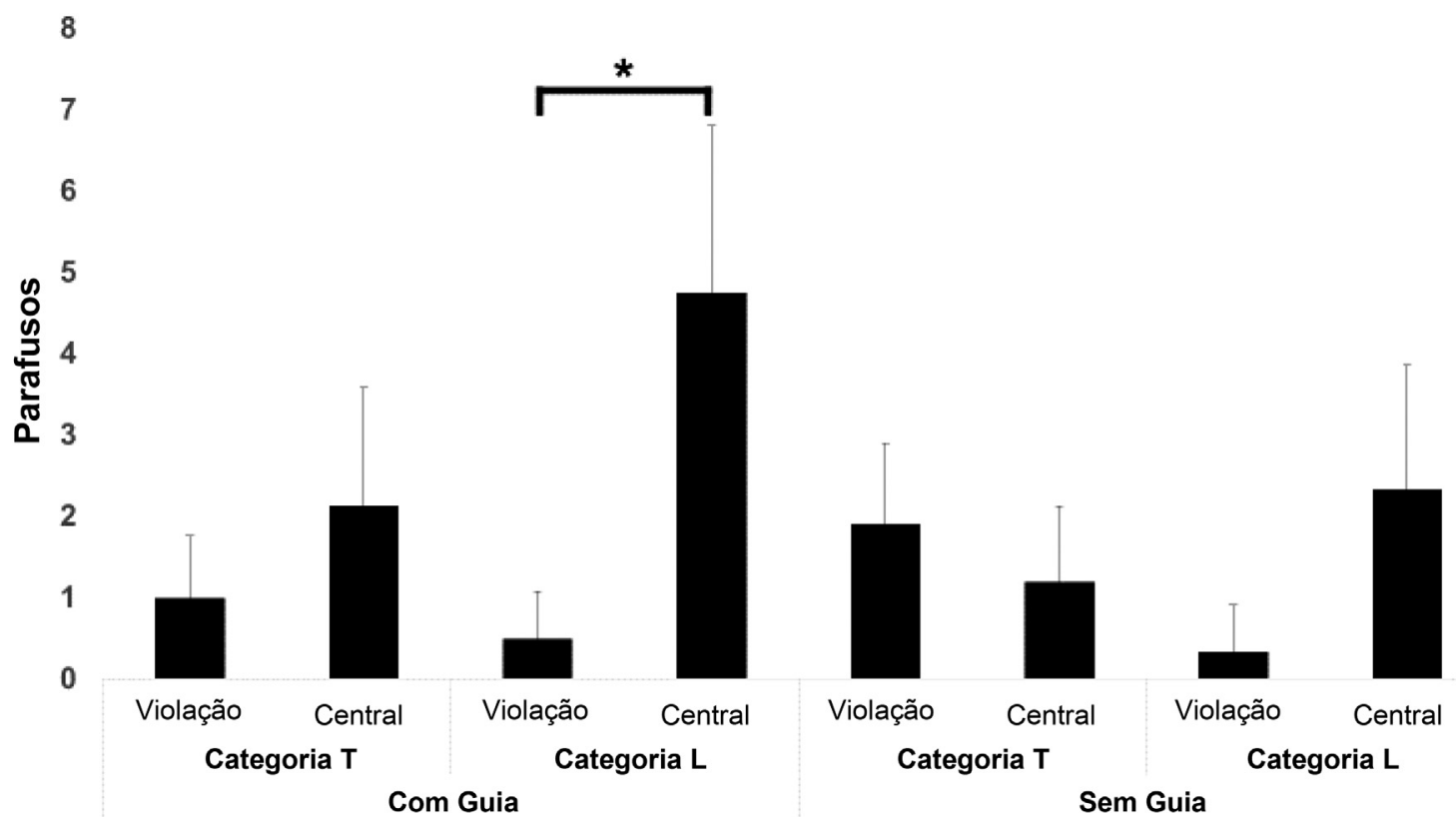

Fig. 6 Precisão do posicionamento dos parafusos pediculares com e sem o uso de guias na coluna torácica (categoria T) e lombar (categoria L) conforme a média do número de parafusos por número de níveis tratados em cada segmento vertebral (lombar e torácico). ${ }^{*} p \leq 0,05$.

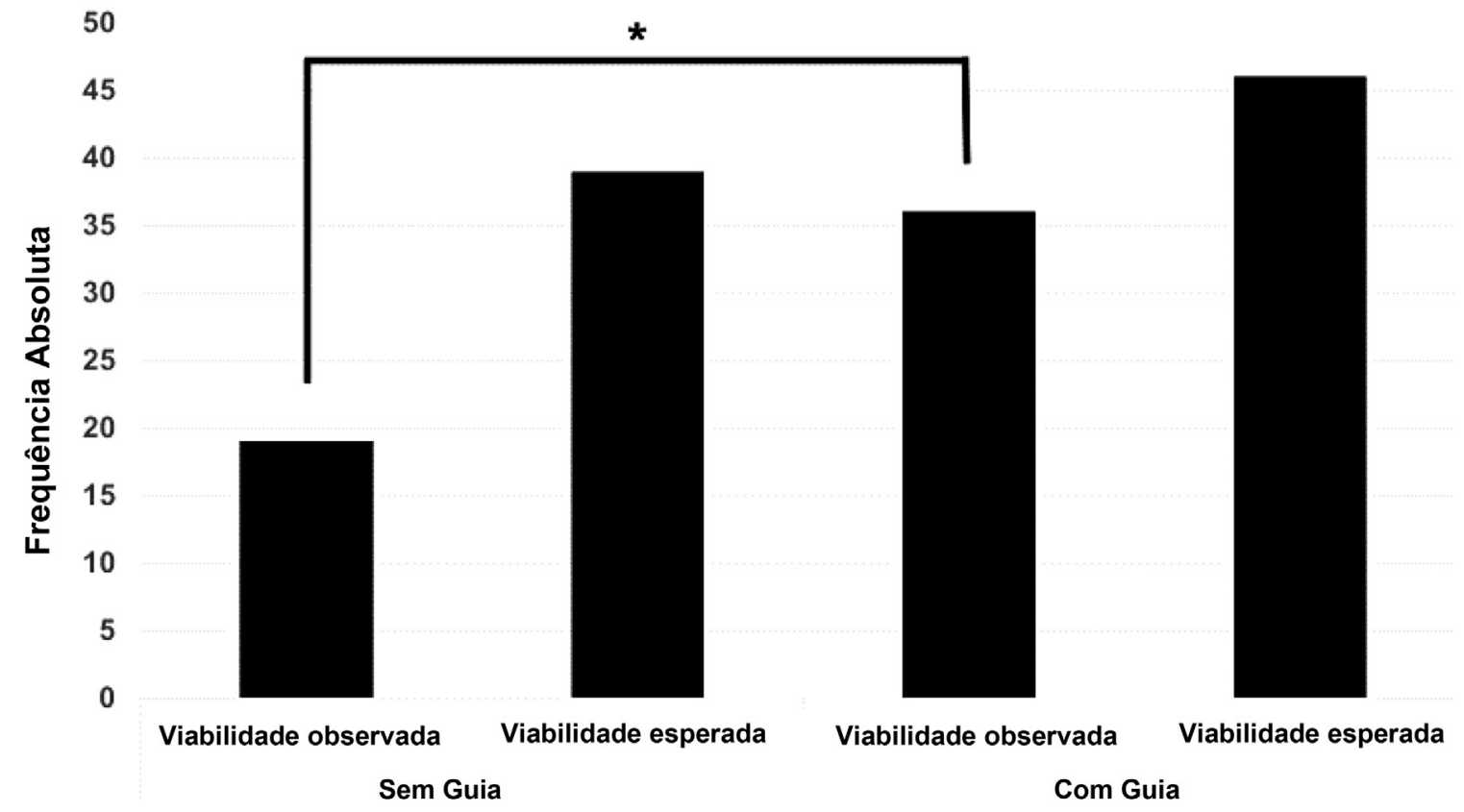

Fig. 7 Viabilidade técnica considerando todos os pedículos instrumentados com e sem o uso de guias. Frequência absoluta: frequência em número absoluto de pedículos tratados. ${ }^{*} p=0,0089$.

tempo de uso da radioscopia variou de 180,3 a 207,2 segundos (média $=190 \pm 16,23$ ).

Não houve complicações operatórias e pós-operatórias, como aumento de sangramento ou lesões neurológicas, nem alterações no potencial motor ou sensorial durante o monitoramento neurofisiológico intraoperatório.

\section{Discussão}

A impressão 3D foi idealizada por Hall ${ }^{20}$ em 1986. A partir daí, a técnica foi aprimorada e introduzida como ferramenta auxiliar nas cirurgias, principalmente de coluna. ${ }^{19} \mathrm{Na}$ cirurgia da coluna vertebral, a impressão 3D tem sido usada na produção de modelos anatômicos, guias e implantes cirúrgicos. ${ }^{20}$

O objetivo do presente estudo foi desenvolver um protótipo de guia personalizada e avaliar seus resultados na confecção do orifício piloto nos pedículos das vértebras torácicas e lombares de pacientes com deformidade espinhal.

A interpretação dos nossos resultados mostra a melhora da cirurgia com a utilização do protótipo, ajustando e corrigindo os problemas encontrados e aumentando sua viabilidade técnica após as cirurgias realizadas. A composição sintética 
120

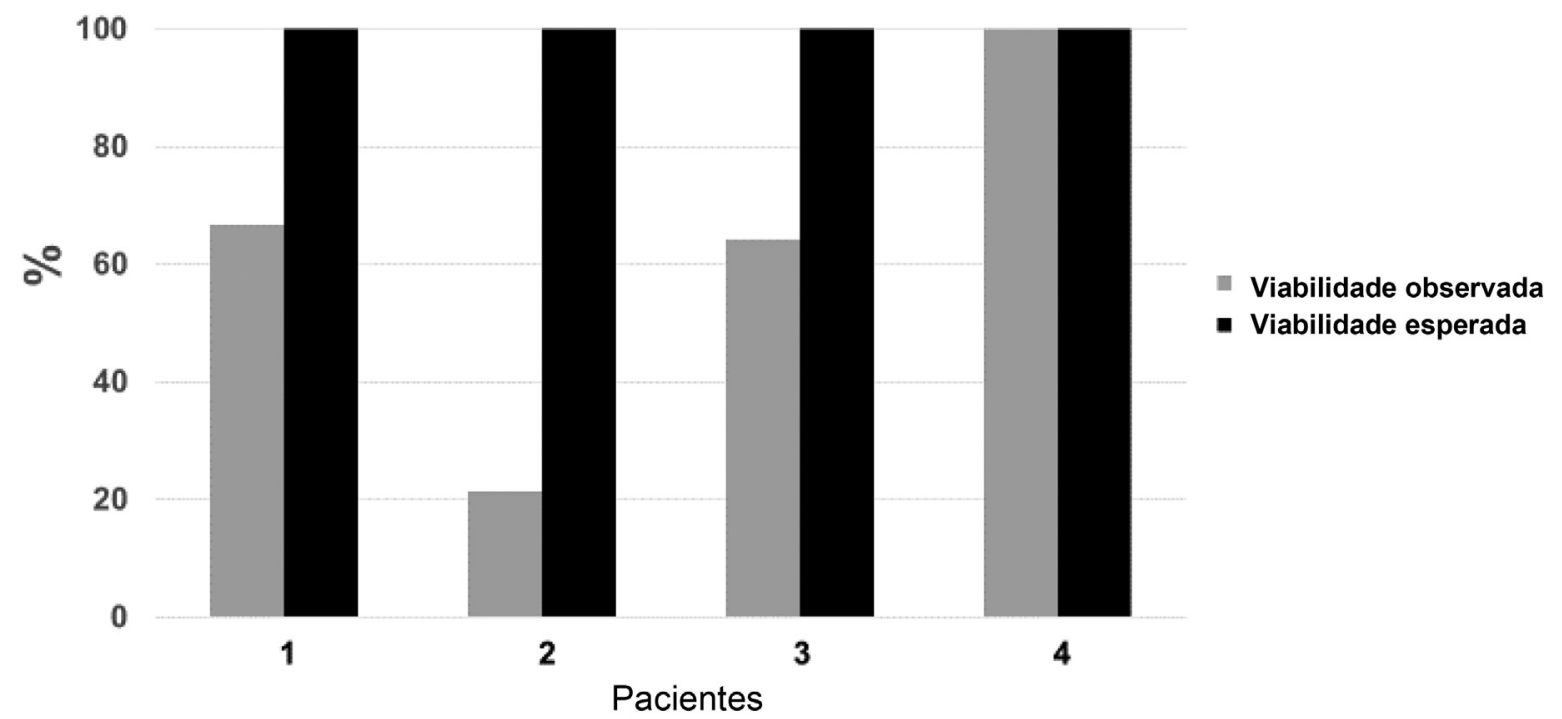

Fig. 8 Viabilidade técnica do uso de guias para cada paciente operado em frequência relativa (percentual).

da guia, seu mecanismo de fixação aos elementos posteriores da vértebra e a melhor adaptação dos instrumentos para preparo do pedículo dentro da guia foram as principais alterações realizadas. Além disso, problemas relacionados à viabilidade técnica do uso da guia foram mais frequentes nos pedículos das vértebras torácicas.

Os problemas relacionados ao encaixe das guias nas vértebras de pacientes com escoliose rígida e alto valor angular também foram relatados por Liu et al. ${ }^{21} \mathrm{O}$ contato mais significativo da guia com a superfície posterior da vértebra aumenta a estabilidade da guia no preparo do orifício piloto; assim, as guias devem ser personalizadas para cada vértebra. Essa observação corrobora os relatos de Berry et al. ${ }^{22}$ acerca da imprecisão das guias para vários níveis.

O encaixe da guia na face vertebral posterior exigiu ampla dissecção e rebatimento das partes moles inseridas nas vértebras. Esse fator também foi apontado como essencial para o encaixe da guia nas vértebras, ${ }^{21}$ e poderia ser apontado como desvantagem da utilização deste tipo de guia em procedimentos de menor extensão. Nas deformidades, porém, a ampla exposição da vértebra e o rebatimento de partes moles são necessárias e não representam uma desvantagem do uso da guia.

O uso de guias aumentou a precisão da colocação do parafuso em relação ao grupo no qual a guia não foi utilizada devido à inviabilidade técnica e ao modelo usado para auxiliar o preparo do orifício piloto. Em pacientes com deformidade espinhal, a taxa de mau posicionamento do parafuso varia de 3 a $44,2 \%$, e a taxa de complicações neurológicas varia de 0 a $0,9 \%{ }^{3,13,16,23-28}$ Os pedículos da coluna torácica e a concavidade da curva apresentam os maiores percentuais de mau posicionamento. ${ }^{3,13,16,23-28}$ Os resultados do presente estudo corroboram os relatos da literatura, e os pedículos da região torácica apresentam o maior índice de mau posicionamento. Porém, apesar do posicionamento descentralizado em 10 pedículos ( 8 torácicos e 2 lombares), não houve danos às estruturas adjacentes ao pedículo ou necessidade de reposicionamento ou retirada de implantes em qualquer paciente.

A precisão do uso de guias nos pedículos torácicos foi de $68 \%$, sendo superior aos resultados do grupo no qual a guia não foi utilizada, evidenciando seus benefícios no preparo do orifício piloto.

De fato, a curva de aprendizado e o desenvolvimento do protótipo da guia devem ser considerados na análise de nossos resultados. Os resultados referentes à precisão no último paciente operado mostraram a alta viabilidade técnica e a precisão próxima à análise anatômica 3D realizada no préoperatório.

A utilização do protótipo de guia personalizada permitiu a redução do tempo de fluoroscopia e, consequentemente, da exposição intraoperatória a radiação. A exposição do cirurgião durante a colocação dos parafusos pediculares é 10 a 12 vezes maior em comparação com outros procedimentos fora da coluna. ${ }^{29,30}$ A dose de radiação intraoperatória em cirurgias para correção de deformidades vertebrais é de $\sim 7,05 \mathrm{mSv}$. Aqui, observamos valores menores, de 4,35 a 6,32 mSv $(5,17 \pm 0,72 \mathrm{mSv})$, indicando menor exposição intraoperatória a radiação. No entanto, a comparação ideal implicaria na análise de grupos semelhantes, o que não foi possível devido à semelhança das amostras heterogêneas; assim, o valor comparativo só pode ser usado como referência.

Embora a técnica de preparo do orifício piloto e inserção dos parafusos pediculares sem o auxílio de imagens ou dispositivos seja considerada segura e tenha precisão aceitável, o uso de guias pode aumentar a eficiência e reduzir a exposição intraoperatória a radiação. A utilização de guias, associada ao conhecimento e experiência do cirurgião, pode tornar o procedimento mais seguro e preciso e diminuir a exposição intraoperatória a radiação. Os resultados aqui apresentados se referem apenas ao desenvolvimento do protótipo da guia, indicando que esse dispositivo pode 

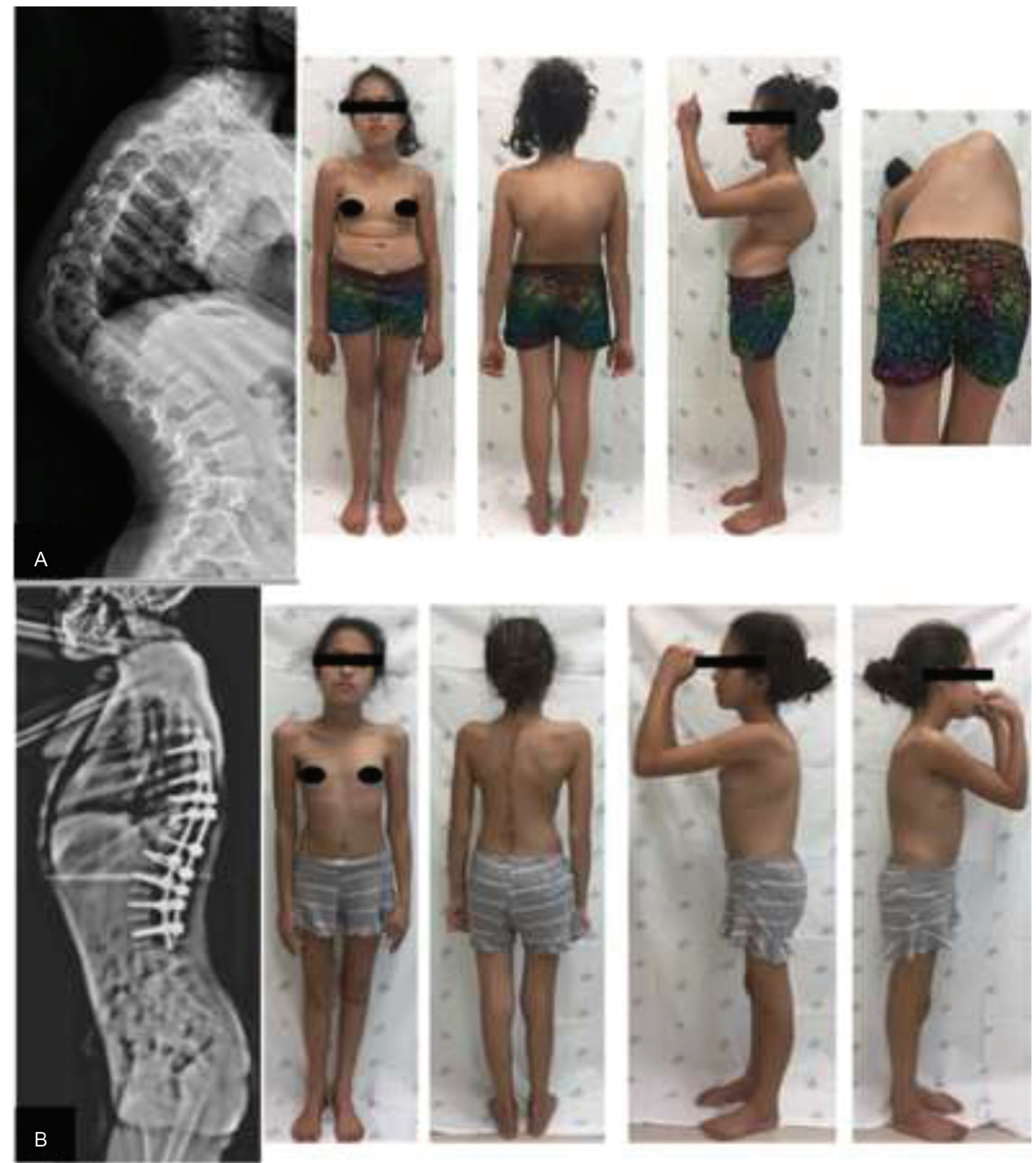

Fig. 9 Imagens radiográficas e clínicas pré (A) e pós-operatórias (B) de um paciente com cifoescoliose congênita (paciente 3) em quem a guia personalizada foi utilizada.

auxiliar a realização de cirurgias de coluna nas quais o pedículo vertebral é o local de ancoragem do implante.

\section{Conclusão}

O uso de guias para preparo do orifício piloto nos pedículos vertebrais de pacientes com deformidade da coluna vertebral foi seguro, melhorou a precisão dos parafusos pediculares e reduziu a intensidade da radiação intraoperatória. Essa tecnologia tem grande potencial clínico, permitindo a colo- cação de parafusos pediculares de forma mais segura, mais precisa, e com menor exposição intraoperatória a radiação.

\section{Contribuição dos Autores}

(I) Concepção e delineamento experimental: Teixeira K.O., Defino H.L.A.; (II) Suporte administrativo: nenhum; (III) Fornecimento de materiais de estudo ou pacientes: todos os autores; (IV) Coleta e preparo de dados: Teixeira K.O., Matos T.D., Fleury R.B.C.; (V) Análise e interpretação dos dados: Teixeira K.O., Bergamaschi J.P.M., Defino H.L.A.; 
(VI) Redação do manuscrito: todos os autores; (VII) Aprovação final do manuscrito: todos os autores.

\section{Suporte Financeiro}

Este trabalho teve suporte do Instituto de Pesquisa e Ensino Home, Brasília, DF, Brasil.

\section{Conflitos de Interesse}

Os autores declaram não haver conflitos de interesse.

\section{Agradecimentos}

Agradecemos à empresa CPMH Produtos Médicos Hospitalares pelo fornecimento do material usado nas cirurgias e suporte científico.

Agradecemos também a Camila Cardador pelo suporte científico e ao professor Walter Krause Neto pela ajuda no preparo final do presente manuscrito.

\section{Referências}

1 Dobbs MB, Lenke LG, Kim YJ, Kamath G, Peelle MW, Bridwell KH. Selective posterior thoracic fusions for adolescent idiopathic scoliosis: comparison of hooks versus pedicle screws. Spine 2006;31(20):2400-2404

2 Webb JK, Burwell RG, Cole AA, Lieberman I. Posterior instrumentation in scoliosis. Eur Spine J 1995;4(01):2-5

3 Boos N, Webb JK. Pedicle screw fixation in spinal disorders: a European view. Eur Spine J 1997;6(01):2-18

4 Maruyama T, Takeshita K. Surgical treatment of scoliosis: a review of techniques currently applied. Scoliosis 2008;3:6

5 Takeshita K, Maruyama T, Murakami M, et al. Correction of scoliosis using segmental pedicle screw instrumentation versus hybrid constructs with hooks and screws. Stud Health Technol Inform 2006;123:571-576

6 Kim YJ, Lenke LG, Kim J, et al. Comparative analysis of pedicle screw versus hybrid instrumentation in posterior spinal fusion of adolescent idiopathic scoliosis. Spine 2006;31(03):291-298

7 Bullmann V, Liljenqvist UR, Schmidt C, Schulte TL. [Posterior operative correction of idiopathic scoliosis. Value of pedicle screws versus hooks]. Orthopade 2009;38(02):198-200, 202-204

8 Merc M, Recnik G, Krajnc Z. Lumbar and sacral pedicle screw placement using a template does not improve the midterm pain and disability outcome in comparison with free-hand method. Eur J Orthop Surg Traumatol 2017;27(05):583-589

9 Abul-Kasim K, Ohlin A. The rate of screw misplacement in segmental pedicle screw fixation in adolescent idiopathic scoliosis. Acta Orthop 2011;82(01):50-55

10 Pan Y, Lü GH, Kuang L, Wang B. Accuracy of thoracic pedicle screw placement in adolescent patients with severe spinal deformities: a retrospective study comparing drill guide template with freehand technique. Eur Spine J 2018;27(02):319-326

11 Esses SI, Sachs BL, Dreyzin V. Complications associated with the technique of pedicle screw fixation. A selected survey of ABS members. Spine 1993;18(15):2231-2238, discussion 2238-2239

12 Halm H, Niemeyer T, Link T, Liljenqvist U. Segmental pedicle screw instrumentation in idiopathic thoracolumbar and lumbar scoliosis. Eur Spine J 2000;9(03):191-197

13 Hamill CL, Lenke LG, Bridwell KH, Chapman MP, Blanke K, Baldus C. The use of pedicle screw fixation to improve correction in the lumbar spine of patients with idiopathic scoliosis. Is it warranted? Spine 1996;21(10):1241-1249

14 Panjabi MM, O'Holleran JD, Crisco JJ 3rd, Kothe R. Complexity of the thoracic spine pedicle anatomy. Eur Spine J 1997;6(01):19-24

15 Liljenqvist UR, Halm HF, Link TM. Pedicle screw instrumentation of the thoracic spine in idiopathic scoliosis. Spine 1997;22(19): 2239-2245

16 Lonstein JE, Denis F, Perra JH, Pinto MR, Smith MD, Winter RB. Complications associated with pedicle screws. J Bone Joint Surg Am 1999;81(11):1519-1528

17 Cordemans V, Kaminski L, Banse X, Francq BG, Detrembleur C, Cartiaux O. Pedicle screw insertion accuracy in terms of breach and reposition using a new intraoperative cone beam computed tomography imaging technique and evaluation of the factors associated with these parameters of accuracy: a series of 695 screws. Eur Spine J 2017;26(11):2917-2926

18 Upendra B, Meena D, Kandwal P, Ahmed A, Chowdhury B, Jayaswal A. Pedicle morphometry in patients with adolescent idiopathic scoliosis. Indian J Orthop 2010;44(02):169-176

19 Kong X, Tang L, Ye Q, Huang W, Li J. Are computer numerical control (CNC)-manufactured patient-specific metal templates available for posterior thoracic pedicle screw insertion? Feasibility and accuracy evaluation. Eur Spine J 2017;26(11): 2927-2933

20 Cecchinato R, Berjano P, Zerbi A, Damilano M, Redaelli A, Lamartina $C$. Pedicle screw insertion with patient-specific 3D-printed guides based on low-dose CT scan is more accurate than free-hand technique in spine deformity patients: a prospective, randomized clinical trial. Eur Spine J 2019;28(07):1712-1723

21 Liu K, Zhang Q, Li X, et al. Preliminary application of a multilevel 3D printing drill guide template for pedicle screw placement in severe and rigid scoliosis. Eur Spine J 2017;26(06): 1684-1689

22 Berry E, Cuppone M, Porada S, et al. Personalised image-based templates for intra-operative guidance. Proc Inst Mech Eng $\mathrm{H}$ 2005;219(02):111-118

23 Kosmopoulos V, Schizas C. Pedicle screw placement accuracy: a meta-analysis. Spine 2007;32(03):E111-E120

24 Cotrel Y, Dubousset J, Guillaumat M. New universal instrumentation in spinal surgery. Clin Orthop Relat Res 1988;227(227): 10-23

25 West JL III, Ogilvie JW, Bradford DS. Complications of the variable screw plate pedicle screw fixation. Spine 1991;16 (05):576-579

26 Suk SI, Kim WJ, Lee SM, Kim JH, Chung ER. Thoracic pedicle screw fixation in spinal deformities: are they really safe? Spine 2001;26 (18):2049-2057

27 Youkilis AS, Quint DJ, McGillicuddy JE, Papadopoulos SM. Stereotactic navigation for placement of pedicle screws in the thoracic spine. Neurosurgery 2001;48(04):771-778, discussion 778-779

28 Gertzbein SD, Robbins SE. Accuracy of pedicular screw placement in vivo. Spine 1990;15(01):11-14

29 Rampersaud YR, Foley KT, Shen AC, Williams S, Solomito M. Radiation exposure to the spine surgeon during fluoroscopically assisted pedicle screw insertion. Spine 2000;25(20): 2637-2645

30 Villard J, Ryang YM, Demetriades AK, et al. Radiation exposure to the surgeon and the patient during posterior lumbar spinal instrumentation: a prospective randomized comparison of navigated versus non-navigated freehand techniques. Spine 2014;39 (13):1004-1009 